\title{
INVESTIGACIÓN
}

Recibido: 29/05/2021 --- Aceptado: 01/07/2021 --- Publicado: 03/01/2022

\section{HÁBITOS DE CONSUMO DE MODA A TRAVÉS DE INFLUENCERS EN LA GENERACIÓN Z}

\section{Fashion consumption habits through influencers on Generation Z}

Davinia Martín Critikián: Universidad CEU San Pablo. España. davinia.martincritikian@ceu.es

María Solano Altaba: Universidad CEU San Pablo. España. msolano@ceu.es

José Francisco Serrano Oceja: Universidad CEU San Pablo. España. pserrano@ceu.es

\section{Cómo citar el artículo:}

Martín Critikián, D., Solano Altaba, M., y Serrano Oceja, J. F. (2022). Hábitos de consumo de moda a través de influencers en la Generación Z. Vivat Academia. Revista de Comunicación, 155, 39-68. http:// doi.org/10.15178/va.2022.155.e1387

\section{RESUMEN}

En plena crisis global, surge la primera generación de jóvenes que ha nacido, se ha educado $\mathrm{y}$ ha socializado desde su inicio con internet y las redes sociales. La irrupción en la sociedad de la generación Z, los jóvenes nacidos entre los años 1994 y 2010, ha hecho que haya que replantearse como poder captar su atención, ya que son jóvenes que viven en una dinámica de inmediatez, defienden la inclusión y son protagonistas de la economía colaborativa y de la diversidad. El presente trabajo analiza cuales son los hábitos de consumo de esta generación, teniendo como objetivo principal, analizar como los influencers han contribuido en los hábitos de consumo. La investigación que se ha llevado a cabo ha sido de carácter cuantitativo, empleando como técnica la encuesta. Esta se ha realizado en el último trimestre del 2020 a 500 jóvenes españoles con una edad comprendida entre los 10 y 25 años. Los resultados obtenidos resaltan que la importancia de los influencers sobre los hábitos de consumo de la Generación Z, gracias a esto, las marcas tratan de establecer relaciones comerciales con ellos con el fin de conectar mejor con su mercado y su

\footnotetext{
${ }^{1}$ Davinia Martín Critikián: Doctora en comunicación, y profesor ayudante doctor de 2011 al 2017 en la Universidad Internacional de La Rioja y del año 2017 a la actualidad en la Universidad CEU San Pablo.
} 
público, para que los influencers transmitan seguridad, transparencia y confianza a cerca de los diferentes productos o servicios que ofrezcan.

PALABRAS CLAVE: Generación Z - Influencers - Hábitos de consumo - Moda Redes Sociales - Sociedad - Medios

\begin{abstract}
A global crisis saw emerge the first generation of youngsters who were born, educated and socialized from the beginning using internet and social networks. The irruption into society of "Generation Z" (young people born between 1994 and 2010), has made it necessary to rethink how to capture their attention, as they are used to living in a world of immediacy, defend inclusion and diversity and are the key stakeholders of the collaborative economy. The present work analyzes the consumption habits of this generation, having as main objective to identify how influencers have contributed to these. The quantitative research that has been carried out has been a survey across 500 young Spaniards (ages of 10 and 25) during the last quarter of 2020. The results obtained highlight the importance of influencers on the consumption habits of Generation Z. Thanks to this, brands try to establish business relationships with influencers, in order to better connect with their market and their audience. The objective of the brands is using influencers to transmit security, transparency and trust about the different products or services they offer.
\end{abstract}

KEY WORDS: Generation Z - Influencers - Consumer habits - Fashion - Social Networks - Society - Media.

\title{
HÁBITOS DE CONSUMO DA MODA ATRAVÉS DE INFLUENCERS NA GERAÇÃO Z.
}

\section{RESUMO}

Em plena crise global, surge a primeira geração de jovens que nasceu, se educou e socializou desde seus começos com a internet e as redes sociais. A irrupção na sociedade da geração Z, os jovens nascidos entre os anos 1994 e 2010, tem feito que seja preciso pensar a forma de captar sua atenção, já que são jovens que vivem uma dinâmica de inmediatez, defendem a inclusão e são protagonistas da economia colaborativa e diversidade. O presente trabalho analisa quais são os hábitos de consumo desta geração, tendo como objetivo principal, analisar como os influencers contribuíram nos hábitos de consumo. A pesquisa que foi feita foi de caráter quantitativo, empregando a técnica de questionários. Esta foi realizada no último trimestre do ano de 2020 a 500 jovens da Espanha, com idades entre os 10 e 25 anos. Os resultados obtidos ressaltam a importância dos influencers sobre os hábitos de consumo da geração $Z$, em consequência, as marcas tentam estabelecer relações comerciais com eles com a finalidade de conectar melhor seu mercado e seu público, 
para que os influencers transmitam segurança, transparência e confiança sobre os diferentes produtos e serviços que oferecem.

PALAVRAS CHAVE Geração Z - Influencers - Hábitos de consumo - Moda - Redes Sociais - Sociedade - Meios de comunicação

\section{INTRODUCCIÓN}

Los jóvenes nacidos entre 1995 y el 2010 son una generación que han nacido en un contexto de Internet donde las pantallas y las redes sociales forman parte del día a día de las personas que les rodean, por ello han aprendido desde el inicio a interactuar con ellas y les ha generado cierta dependencia de estas. esta generación tiene una serie de características como son la creatividad, la capacidad de hacer varias cosas al mismo tiempo o en diferentes pantallas, lo cual ha propiciado un cambio de enfoque en el mundo del marketing digital, así como en los creadores de contenido en redes sociales los cuales cuentan con una alta credibilidad para las marcas como prescriptores. (Fernández, 2019).

\section{OBJETIVOS}

La presente investigación tiene como objetivo general analizar cuales son los hábitos de consumo de moda a través de influencers en la generación Z. Para la consecución del mismo, se proponen una serie de objetivos específicos como son:

1. Estudiar el contexto social en el que está la generación $\mathrm{z}$

2. Determinar los hábitos de consumo de los jóvenes nacidos entre el 1995 y el 2010.

3. Investigar cómo afecta el papel de los influencers a dicha generación

\section{METODOLOGÍA}

Para llevar a cabo la investigación de manera adecuada y lograr los objetivos planteados, se ha llevado a cabo por un lado una investigación documental en base a fuentes primarias y secundarias analizando y consultando diferentes páginas web, libros de texto, revistas, repositorios y tesis doctorales. Y por otro, una investigación de tipo cuantitativo empleando como herramienta una encuesta contestada por 500 jóvenes.

\subsection{Fundamentación teórica}

\subsubsection{Contexto social}

Numerosos conceptos pueden introducirse como válidos a la hora de definir qué es la Sociedad de la Información ya que dependerá de las fuentes consultadas:

Nuevo sistema tecnológico, económico y social. Una economía en la que el incremento de la productividad no depende del incremento cuantitativo de los 
factores de producción (capital, trabajo, recursos naturales), sino de la aplicación de conocimiento e información a la gestión, producción y distribución, tanto en los procesos como en los productos." (Castell, 2012, p.102)

Estadio económico y social cuyas acciones de supervivencia y desarrollo están caracterizadas por la capacidad potencial de sus miembros (personas y organizaciones) de hacer un uso evolutivo (extensivo, intensivo y estratégico) de las TIC para interconectarse en red entre ellas de cómo convergente, ubicuo, instantáneo y multimedia." (Finquelievich, 2008, p.45)

Sociedad de la Información es una fase de desarrollo social y caracterizada por la capacidad de sus miembros (ciudadanos, empresas y Administración Pública) para obtener y compartir cualquier información, instantáneamente, desde cualquier lugar y en la forma que se prefiera" (Castell, 2012, p.32)

A través de las diversas definiciones que encontramos sobre la materia, se puede observar que a pesar de su breve variación entre unas y otras, todas coinciden en el hecho de la influencia que la Tecnología realiza sobre los pilares de una nueva Sociedad. Sin embargo, este tipo de Sociedad lleva desarrollándose ya desde los años sesenta, cuando el modelo de Sociedad Industrial fue quedando obsoleto en cuanto a claves económicas se refiere para dejar paso a un nuevo comienzo de sociedad en el que el procesamiento y manejo de la Información toman un papel relevante respecto al control y optimización de los procesos industriales.

Muchos de los cambios producidos en la estructura industrial y las relaciones sociales son parecidos a los que previamente modificaron cambios sociales como la Revolución Industrial, al pasar de una sociedad de fundamento esencialmente agrario a una sociedad industrializada. No obstante, hay un factor diferencial que se introduce con este nuevo tipo de Sociedad de la Información y va implícito en su nombre.

Anteriormente, cada persona disponía de sus propios almacenes de conocimiento, que siempre se intercambiaban y realizaban de una forma selectiva y rudimentaria. En este caso en particular lo peculiar se da en la posibilidad ilimitada de acceso a la información generada por otros seres, así como por uno mismo.

La Sociedad de la Información avanza a un ritmo frenético y este avance hace que la información a la que estamos expuestos pueda llegar a desbordar al usuario.

Es aquí donde comienzan a surgir las críticas a esta nueva forma de sociedad puesto que la continua evolución provoca que se introduzcan infinitas posibilidades. Con todo ello hay que tener en cuenta que, a pesar de no tener una estructura definida, dada su versatilidad y cambio constante a lo largo del tiempo, la Sociedad de la Información se configura de forma tal que dentro de su propio "caos" de información pueda llegar a encontrar un sentido en su conjunto. 
Para la definición de la Sociedad de la Información encontramos diversas opiniones y definiciones, pero sin embargo todos coinciden en la importancia de las nuevas tecnologías para su desarrollo. Tienen un papel fundamental dentro de todo esto el usuario, entorno, medio e infraestructuras que serán común en todas las posiciones adoptadas para el desarrollo de esta Sociedad.

\subsubsection{Elementos principales de la sociedad de la información}

Son cuatro los elementos o características en las que podemos dividir y desglosar el concepto de Sociedad de la Información.

\section{1) Usuarios:}

Por consumidores o usuarios debemos entender según el artículo 1, párrafo 2o de la Ley 19-7-1984, núm. 26/1984, General para la Defensa de los Consumidores y Usuarios:

A los efectos de esta Ley, son consumidores o usuarios las "personas físicas o jurídicas que adquieren, utilizan o disfrutan como destinatarios finales, bienes muebles e inmueble, productos, servicios, actividades o funciones, cualquiera que sea la naturaleza pública o privada, individual o colectiva de quienes los producen, facilitan, suministran o expiden". (LGDCU, 1984)

Las Nuevas Tecnologías, además de facilitar la adquisición de estos nuevos productos y servicios relacionados con ellas misma, nos ofrecen un nuevo espacio a los usuarios, Internet, donde poder encontrar servicios y bienes. Sin embargo, a tales efectos las personas se convierten en pacientes de la Sociedad de la Información, ya que vamos dejando un rastro de información que es fácil de conseguir y procesar.

\section{2) Entorno:}

En este caso tratamos todos aquellos factores que influencien tanto social como económicamente a favor de la implantación de la Sociedad de la Información. Esto puede ser la cultura, formación, economía o política. Cabe destacar la importancia que tendrán a la hora del desarrollo futuro de este tipo de Sociedad.

3) Medio:

Es la información, los productos y servicios a los que podemos acceder sin la necesidad de desplazarse a un determinado sitio. Dentro de todos ellos, se diferencian cuatro grupos dependiendo de las necesidades o prestaciones que sean capaces de ofrecer. Medios tangibles, intangibles, de servicios o de infomediación.

Aquellos tangibles e intangibles ofrecerán una misma misión o función al usuario, pues ambos dos serán el opuesto de ellos mismos. Esto quiere decir que los medios 
intangibles serán aquellos que brinden la oportunidad de obtenerlo de manera gratuita, mientras que los tangibles serán aquellos por los cuales debamos pagar.

Otros medios que debemos destacar son los que se dedican a los servicios. Éstos, ocupan su espacio para procurar ayuda y resolver cualquier tipo de acción que pueda llegar a facilitar su desarrollo gracias a ellos.

La infomediación es la última tipología de medios a destacar, trata aquellos medios por los cuales obtenemos información. Esta información la podemos obtener gracias a las estructuras de telecomunicaciones, los servidores o equipos y las redes.

Las infraestructuras de telecomunicaciones van a ser los medios técnicos que hacen posible el acceso a distancia de los contenidos. Estas infraestructuras son de vital importancia para el crecimiento de esta sociedad.

Además, la existencia y desarrollo de la telefonía móvil, ha provocado que la información se encuentre al alcance del usuario en cualquier momento desde prácticamente cualquier lugar. Además el intercambio de información entre usuarios es constante instantáneo.

1. Servidores o Equipos: Es dónde se almacena contenido en origen. Son tanto parte de los terminales como equipos autónomos dedicados exclusivamente a almacenar y contener toda aquella información que se mantiene en red; aquella información que deja huella por parte del usuario.

2. Las redes: Distribuyen los contenidos y pueden ser a su vez de acceso o de transporte. Esto quiere decir que puede conectar a usuarios con los servidores a la vez que transportan grandes cantidades de información de un punto a otro del planetas. Ambas están conectadas entre sí, por lo que no se concibe el uso de una sin la otra. A su vez, el usuario forma parte fundamental de la red pues será el que haga posible que toda la información viaje a través de estas conexiones.

\subsubsection{Sociedad del conocimiento}

La Sociedad del Conocimiento es aquella que establece que la sociedad se caracteriza por una estructura social y económica donde el trabajo se sustituye por el conocimiento. Es decir, el conocimiento sustituye a materias primas, trabajo y al capital como fuente más importante de productividad, crecimiento.

Peter Drucker es el autor principal al que se le atribuye el concepto de la Sociedad del Conocimiento como sociedad post-moderna. Aunque, además de Drucker, este término también se le atribuye a algunos teóricos japoneses. (Szabo, 2005)

Dentro de estas atribuciones, Drucker nos muestra una explicación algo más estructurada sobre la sociedad del conocimiento, explicando su origen al poco de 
finalizar la Segunda Guerra Mundial, y con los sucesivos cambios ideológicos. (Drucker, 1996)

Bell (2001) hizo un estudio pormenorizado sobre la sociedad post-industrial. Este concepto expresó la transición de una economía que produce productos a una economía basada en servicios y cuya estructura profesional está marcada por la preferencia a una clase de profesionales técnicamente cualificados. El conocimiento teórico se ha convertido, según este enfoque, en la fuente principal de innovación y en el punto de partida de los programas políticos y sociales. Este tipo de sociedad está orientado hacia el progreso tecnológico y la evaluación de la tecnología y se caracteriza por la creación de una nueva tecnología intelectual como base de los procesos de decisión. (Krüger, 2006)

Por tanto, la sociedad pasa a ser un tipo de sociedad científica, académica y centrada en los servicios que se posicionaban en contra de la sociedad industrial, caracterizada por el conocimiento experimental, el dominio de sectores industriales, actividades manuales y el conflicto entre capital y trabajo.

Sin embargo, como menciona Krüger, es importante mantener una postura algo escéptica respecto a lo que Sociedad del Conocimiento representa, pues al ser un concepto tan general queda en el aire un poco su validez o uso exclusivo para la definición de una Sociedad.

La evolución de la sociedad a lo largo del tiempo se basa en el conocimiento, pues sin este no habría posibilidad de evolución. Con ello, debemos mantener nuestra postura desde un punto de vista menos literal en cuanto a Sociedad del conocimiento se refiere, a uno más conceptual sobre nuestra realidad. De otro modo, estaríamos dando por hecho que hemos alcanzado la cima del conocimiento, o incluso estaríamos negando la existencia del conocimiento en otras sociedades anteriores.

La Sociedad del Conocimiento se caracteriza entonces por un uso destacado de la información gracias a las nuevas tecnologías, que van más allá de la comunicación entre las personas, creando conocimientos nuevos. Ello se produce por un fuerte progreso en innovación. (Cornellá, 1998)

Como se mencionaba previamente en la definición de Sociedad de la Información, los usuarios manejan abundante información que hoy día resulta desbordante. Es en esta fase en la que estos usuarios deberán adquirir nuevas habilidades para ser capaces de prosperar en un mundo lleno de continuas alteraciones. Esto afecta desde a los sistemas educativos, hasta a los mercados laborales.

No todos los países tienen la oportunidad de acceder a economías fundadas en el conocimiento, e incluso en muchos de los países avanzados que si tienen esta capacidad existen sectores de la sociedad donde el acceso al saber es un factor de exclusión. 
La privatización de las bases del conocimiento supone una barrera para llegar a adquirir nuevos conocimientos, impidiendo de este modo el desarrollo humano. (Farnos, s.f.)

\subsubsection{Inmersión en la sociedad del saber}

A lo largo de la Historia han surgido instituciones dedicadas exclusivamente a la difusión y creación del conocimiento. El conocimiento ha sido un elemento clave a lo largo de la historia en la creación de las diferentes sociedades que ha ido desarrollando el ser humano.

Como hemos mencionado con anterioridad, no existe una sociedad sin un conocimiento previo que pueda aplicarse al desarrollo de la misma. Por tanto, para avanzar de una sociedad a otra es indispensable la parte de creación e innovación, inventar nuevos conocimientos e ideas que materialicen luego en productos que produzcan un desarrollo económico y de bienestar a la sociedad, en definitiva hablamos de Evolución.

Debemos tener en cuenta:

1. Incremento de Conocimientos:

El aspecto central de un punto constituido por una aceleración del ritmo de creación y acumulación del conocimiento sin precedentes. Esto es debido a una fuerte intensidad de progreso científico y tecnológico, que hace que los conocimientos estén alcanzando un ritmo frenético, donde las múltiples consecuencias y retos tanto económicos como sociológicos hacen que el avance llegue en un momento álgido de la historia de estos.

Todo ello ayudado por unas comunidades de conocimiento, redes de individuos, cuyo objetivo es compartir e intercambiar conocimientos para el desarrollo. Al realizar este tipo de actividades, estas sociedades están transformando la sociedad. (Martínez et al., 2006)

2. Capital Intangible:

"Los Historiadores del crecimiento explican que los recursos naturales y su abundancia han perdido gran parte de su capacidad de explicar las disparidades de productividad y de crecimiento entre los países. En cambio, la mejora de la calidad del equipo físico y del capital humano representan una mejor explicación, es decir, la creación de nuevos conocimientos y de nuevas ideas y su incorporación al equipo físico y a las personas." (OCDE, 1999)

Esto quiere decir que gran parte del capital intangible está constituido en actividades que hagan que esos nuevos conocimientos se establezcan y asienten en la sociedad. Es decir, este capital se desarrollará en capacitación, instrucción, I+D, inversiones todas ellas que den fruto en la producción y 
transmisión de conocimientos tanto anteriormente utilizados como recién adquiridos.

3. Innovación como Actividad Dominante.

La innovación y el progreso son necesidades para el ser humano, ya que es un medio para sobrevivir y prosperar en estas sociedades de economías competitivas y globalizadas. La sociedad avanza ya no solo por el hecho de unos cuantos grupos de personas que captan todo el conocimiento avancen según sus intereses, sino que la sociedad necesita innovadores, personas que vean del progreso una necesidad para el ser humano.

4. Evolución en los instrumentos.

En este apartado tratamos las infraestructuras de telecomunicaciones mencionadas anteriormente para la Sociedad de la Información desde un punto de vista que afecta al conocimiento en sí. Distinguir los diversos tipos de repercusiones de las tecnologías sobre la creación de conocimiento y que han constituido un poderoso sistema de creación de conocimiento. Como por ejemplo la creación en abundancia de información o la posibilidad del tratamiento de bases de datos.

\subsubsection{Características de la sociedad del conocimiento}

Tras haber analizado la introducción y definición sobre lo que afecta y en lo que se fundamenta la Sociedad del Conocimiento, cabe destacar las características principales en las que se podría resumir todo lo mencionado anteriormente. Son:

1. Conversión del Conocimiento en factor crítico para el desarrollo productivo y social. Lo que quiere decir que no se entenderá el desarrollo de la sociedad sin el desarrollo del conocimiento. Por tanto, es imprescindible el hecho de que la sociedad, o parte de ella sea capaz de tener acceso a la información que hará posible el desarrollo de este conocimiento.

2. Fortalecimiento de los procesos de aprendizaje social como medio para asegurar la apropiación social del conocimiento y su transformación en resultados útiles, en donde la educación juega el papel central ya que harán que las generaciones venideras sean capaz de asimilar los conceptos que esta nueva sociedad ha materializado y así poder avanzar. (Foray, 2002)

\subsubsection{Principales diferencias entre ambas sociedades}

Las diferencias que podemos encontrar entre ambos tipos de sociedades no es más que una diferencia de conceptos. En la actualidad denominamos a todos estos cambios sociales, económicos, tecnológicos e industriales de muchas formas, que pueden ir desde la Sociedad en Red, Sociedad de la Información o la propia Sociedad del Conocimiento.

¿Qué diferencia hay entonces entre ambos conceptos? 
Para tratar de explicar utilizaremos un fragmento del artículo escrito por Karsten Krüguer en Revista bibliográfica de Geografía y Ciencias sociales (serie documental de Geo) en la que dice:

La noción de la 'sociedad de la información' se utiliza sobre todo cuando se tratan aspectos tecnológicos y sus efectos sobre el crecimiento económico y el empleo. Esta discusión tiene como punto de partida la consideración de que la producción, la reproducción y la distribución de la información es el principio constitutivo de las sociedades actuales. (Krüger, 1999)

Pero en la reciente discusión europea, tanto en el ámbito de las ciencias sociales como en el ámbito político, se observa que este término es reemplazado por el de la Sociedad del Conocimiento lo que implica un cambio conceptual de la información al conocimiento considerándolo como principio estructurador de la sociedad moderna y resaltando su importancia para la sociedad actual, para los cambios en la estructura económica y en los mercados laborales, para la educación y para la formación.

Otra noción alternativa es la de la sociedad red, noción promovida por Castells. Se trata de un concepto que está situado entre la Sociedad de la Información y la Sociedad del Conocimiento. Castells marca diferencias respecto a la Sociedad de la Información, distinguiendo entre información e informacional. Información, es decir comunicación del conocimiento, ha sido, según Castells:

Fundamental en todas las sociedades (...) En contraste, el término informacional indica el atributo de una forma específica de organización social en la que la generación, el procesamiento y la transmisión de la información se convierten en las fuentes fundamentales de la productividad y el poder, debido a las nuevas condiciones tecnológicas que surgen en este periodo histórico. (Castells, 2000)

Podría llegarse a entender por tanto que la Sociedad del Conocimiento es una consecuencia de la Sociedad de la Información, pues sin la primera de ellas la segunda no existiría, se reduciría a un conocimiento entendido dentro de otro tipo de Sociedad.

La diferencia entre ambos conceptos, es prácticamente inexistente, ya que, la Sociedad del Conocimiento, es una consecuencia de la Sociedad de la Información. (Castells, 2000) Si bien, están muy cerca la una de la otra, una de sus principales diferencias es el uso de la tecnología.

En el caso de la Sociedad de la Información, la tecnología es un elemento fundamental, pues sin ella esta sociedad tal y como se define no existiría, sin embargo para la Sociedad del Conocimiento el hecho del uso de las tecnologías es un componente más para el avance del conocimiento pero no es el elemento determinante de esta sociedad, que interpreta y da sentido a la abundante información recabada. 
La Sociedad del Conocimiento llega a producir un cambio en la Sociedad mucho más allá de lo que se pueda imaginar. Incluye una dimensión de transformación social, cultural, económica, política e institucional, así como una perspectiva más desarrolladora. Por el contrario, la Sociedad de la Información está relacionado con la idea de innovación en las tecnologías y captación de información de los usuarios.

Observamos por tanto que es una evolución más de la sociedad. Sin evolución, sin transformación en todos los ámbitos de la sociedad, el conocimiento no avanza, no desarrolla su potencial, y sin el avance de estos elementos y de la sociedad propia, ninguna nueva sociedad se habría formado.

Es necesaria la aceptación de los conocimientos, el asentamiento en la sociedad de los mismos para poder llegar a entender todo lo que se menciona y así poder innovar y continuar en el proceso evolutivo. Esto no es posible, sin un código que haga que los conocimientos queden plasmados para la fácil compresión de aquellos que van a aceptarlos.

\subsubsection{Procesos de socialización}

El ser humano vive en sociedad, es algo innato y a lo que estamos predestinados al nacer. Sin embargo, para convivir con los demás no solo basta con esto, es necesario aprender todos aquellos conocimientos y habilidades que son necesarios. Es imprescindible un entrenamiento que nos permita la adaptación al medio en el que nacemos. Esto es lo que conocemos como socialización. (Ministerio de Educación, Política social y deporte, s.f.)

\subsubsection{Definición de socialización}

Según la Real Academia de la lengua española se entiende por socialización a "la acción o efecto de socializar" entendiendo por socializar "transferir al estado, o a otro órgano colectivo, algo de propiedad privada, especialmente un servicio o un medio de producción" o como segunda acepción "Extender al conjunto de la sociedad algo limitado antes a unos pocos" (RAE, 2016)

La socialización por tanto es la forma en la que aprendemos a adaptarnos a un medio natural en unas determinadas circunstancias para la integración con otros seres humanos. Es el aprendizaje el que nos hace aptos para un entorno u otro y será el que nos ayudará a no ser excluidos por nuestros semejantes, la sociedad a la que nos adaptemos.

La socialización consiste pues en la adquisición de habilidades, creencias, normas y costumbre de la cultura en la que nos ha tocado vivir.

Estos conocimientos se adquieren de una forma gradual a lo largo de nuestras vidas, de ahí que se denomine a la socialización como un proceso de aprendizaje 
(Calderón, s.f). Un aprendizaje que tardara varios años en ser capaz de completarse hasta que llegue el momento en el que nuestro cuerpo no sea capaz de retener toda la información que nos gustaría o, mejor dicho, que retiene con una lentitud e imperfección mayor a lo que lo hacía previamente.

Por tanto, cabe reseñar que no hay una edad límite para el aprendizaje, aunque es cierto que hay etapas en las que el aprendizaje es mucho mayor que en otras. La capacidad de aprender en la infancia del individuo no es la misma que tiene una persona con 60 años. La infancia es el momento en el que parece que los individuos somos capaces de adquirir la mayor capacidad de conocimientos que el ser humano logra almacenar. Por poner un ejemplo, es más sencillo que un niño consiga adaptarse a la comunicación en diferentes idiomas a que esto mismo lo consiga un adulto (Maestre, s.f). Es consecuencia de esto que decimos por lo que podemos identificar dos tipos de socialización: La socialización primaria y la socialización secundaria.

\subsubsection{Socialización primaria y secundaria}

Como acabamos de mencionar, existen dos tipologías de socialización según las etapas de aprendizaje de conocimientos o de asimilación de conceptos en la que nos encontremos. (Villegas, 2010)

\section{1) Socialización Primaria}

Es la más importante de las etapas de socialización, ya que introduce al sujeto en la sociedad. En sus primeros años de vida irá adquiriendo determinadas pautas y costumbres del grupo social al que tenga más apego.

Estos agentes o grupos sociales suelen ser en sus primeros años de vida la familia, con la que el individuo se convierte en un ser social. El individuo va asimilando los roles y actitudes de las personas que tiene a su alrededor y con las cuales se siente identificado. Es, sin lugar a dudas, el primer paso hacia la creación de una identidad.

La socialización implica una interiorización de roles. Este proceso de aprendizaje de los roles se realiza desde un plano específico a lo general. Sin embargo, este proceso de socialización no deja de ser un acto más emocional que cognitivo.

Del éxito de este aprendizaje depende la formación integral de la persona y su correcta adaptación al entorno. Un entorno adecuado es primordial, de lo contrario puede tener consecuencias irreversibles en la identidad del ser que está tratando de sociabilizarse, generando comportamientos que la sociedad puede rechazar, generando exclusión de la misma. (Prats, 2015)

2) Socialización secundaria 
Es aquella por la cual el individuo conoce otro tipo de patrones y pautas que interiorizará. En este caso hablamos de una evolución/modificación del aprendizaje base del que hablábamos en el apartado anterior.

Es otra optativa para el desarrollo de su identidad y en un estado más avanzado a la socialización primaria. Sin embargo, no va en contra de lo ya aprendido, sino que se intenta ayudar a ello y sumar experiencias que puedan desarrollar una mejor conducta de lo previo. (Villegas,2010)

Estas instituciones formaran el carácter de la persona y harán que su sociabilización se focalice más en unos puntos que en otros. Es quizás la que más tiene que ver con el tema el cual tratamos sobre la Sociedad de la Información y la Sociedad del Conocimiento.

Si el primer proceso socializador ha sido efectivo, este segundo será una continuación del mismo. En caso de no haber sido efectivo el primer proceso socializador por diferentes motivos, este segundo proceso de sociabilización será más complicado para el individuo.

\subsubsection{Agentes}

Todos aquellos individuos que desempeñen la acción de introducir en la sociedad a otro individuo, este será un agente de socialización. Hablamos de personas, grupos, instituciones, cualquier elemento del entorno que forme parte de este proceso.

"Cuando desertamos de la conciencia, del compromiso, de la responsabilidad y del amor que entrañan la concepción de una vida de éste "accidente biológico" tiene como consecuencia la orfandad. La orfandad, entendida de éste, su aspecto más devastador, deja a los hijos sin amor, sin referencia, sin alimento emocional, sin guía ética, sin modelos existenciales, sin nutrientes espirituales. (Sinay, 2007)

Con este texto, señalamos al primero de los agentes más importantes a la hora de intervenir activa y directamente en el proceso de socialización. (Cerón, 2015)

Algunos de estos agentes que indicamos son:

1. La familia:

Es el primer agente y uno de los más importantes, ya que será este el que tenga la capacidad de moldearlo e incluir al individuo de una manera decisiva para una futura sociabilización con el exterior. Es prácticamente el único contacto que tomará antes de entrar en la escuela, una sociabilización que tardará un par de años dependiendo del individuo.

Por tanto, aunque las familias cambien según las culturas, siempre son las encargadas de preparar al nuevo individuo para todo lo que en un futuro pueda llegar a suceder una vez inmerso en la sociedad. 
La huella que ellos dejen será algo prácticamente imborrable, por lo que es necesario que la familia tome consciencia de que lo que ellos hagan repercutirá directamente en el sujeto durante el resto de su existencia.

Como se ha mencionado en el apartado anterior, el que una persona en esta etapa conste del apoyo de familiares o no hará que la persona aporte beneficios o problemas futuros al conjunto de la sociedad.

2. La escuela:

Otro de los ejemplos fundamentales tomados para la definición de la etapa secundaria. Es importante mencionar que es la institución constituida expresamente para la formación y educación de los nuevos miembros de la sociedad. Por lo que no solo refuerza al valor aportado por las familias, sino que desarrolla nuevo conocimiento en el individuo que le ayudarán a su evolución personal.

El individuo debe acudir a este tipo de centros especializados donde se le enseñará a manejar ciertos elementos concretos que le permitan entender la sociedad desde un cierto punto de vista que moldeará no solo su personalidad sino también la capacidad de manejo de los conocimientos más avanzados.

En estos centros, el individuo estará en un continuo aprendizaje, lo cual resulta un doble aspecto socializador, ya que no solo empezará a asimilar conceptos de las diversas materias en se impartan, sino que además adquirirá habilidades diferentes gracias al contacto con otros individuos (trabajos en equipo, respeto de normas, colaboraciones, etc.).

En este caso, es importante mencionar la gran ayuda que este tipo de agente de socialización crea a los individuos, pues son realmente los que harán que pase de un estado muy precario a una socialización bastante avanzada, dependiendo claro está del lugar en el que se encuentre y la zona del mundo en el que se le imparta esto (desigualdad ya tratada anteriormente). (Villegas, 2010)

3. El grupo de iguales:

Estos individuos son fundamentales para el desarrollo de socialización de un sujeto, pues le darán una visión totalmente diferente a la que sus familiares o la escuela pueda mostrarles.

Ya se mencionaba en el agente de socialización escuela el contacto con otros individuos a través de trabajos, colaboraciones y demás. No obstante, es importante destacar la acción de estos individuos que muchas veces podrán influir incluso más que los propios seres de una familia. (Villegas, 2010)

4. Los medios de comunicación: 
Son fundamentales también pues tendrán contacto directo y continuado con la Sociedad de Información y Sociedad del Conocimiento. Si bien todo lo tratado está relacionado, éstos están en directa conexión con el individuo.

Es importante destacar, que previamente hemos tratado estos elementos de una forma en la que hacía hincapié en el contacto con los individuos. A pesar de ello, ahora nos centraremos en la capacidad de influencia que tienen estos agentes/elementos en la socialización del individuo, cuya mente está creciendo socialmente.

Es importante ser consciente que las tecnologías son un elemento que pueden llegar a perjudicar a la capacidad social de toda persona, ya sea niño o adulto, que la utilice.

En la actualidad el proceso socializador está en constante evolución debido a las posibilidades que están aportando las nuevas tecnologías. Poder comunicarte con cualquier parte del mundo o la facilidad para adquirir información de cualquier tipo influencia la evolución personal que tiene el individuo.

Existe la posibilidad de captar conocimientos a los que hasta el momento el individuo no tenía opción. Esto es debido por un lado por los cambios producidos por las nuevas tecnologías y por otro por la evolución de la comunicación a nivel mundial, forma parte del proceso de globalización.

Pero no todo es bueno en esta innovación en el proceso sociabilizador, puesto que la información puede usarse por individuos con intenciones negativas y podrían provocar la interiorización de comportamientos irreversibles en los individuos.

\subsubsection{Cambios sociales}

"Con la invención de los ordenadores, la humanidad por primera vez estuvo en condiciones de fabricar un portador de información interactivo. Hasta ese momento, el ser humano era el único portador de información interactivo, porque era capaz de aplicar la información almacenada para contestar preguntas y resolver problemas. Apoyándose en la más moderna tecnología, ahora se pueden producir industrialmente máquinas que también van a disponer de semejante capacidad interactiva. Justamente por esta razón, la informática y la tecnología de las comunicaciones constituyen pilares básicos de la Sociedad de la Información" (Gómez, 1999)

Las nuevas tecnologías, como el uso de ordenadores y sistemas operativos portadores de información son los medios mediante los cuales están variando las relaciones que el ser humano tiene en esta nueva sociedad. 
"Internet no sólo es un nuevo medio de información y comunicación, sino que, junto con otros sistemas tecnológicos periféricos (multimedia, infojuegos, realidad virtual, etc.), configura un nuevo espacio social, electrónico, telemático, digital, informacional y reticular, al que cabe denominar "tercer entorno". El tercer entorno se superpone a los otros dos, el campo y la ciudad (physis y polis), y genera profundas transformaciones en la vida humana y social.

\subsubsection{Consumidor como emisor de contenidos}

Según Sequeiros, puente López y Fernández en 2016, gracias al surgimiento de las webs colaborativas y de contenido como las wikis o los blogs, provoco que la personas pudieran convertirse en elaboradores y generadores de contenido, sin necesidad de tener formación previa al respecto.

Este ir i venir de información instaura la cultura digital permitiendo que internet forme parte de la vida cotidiana y que las tecnologías de la información y la comunicación se conviertan en herramientas para desarrollar actividades como las compras y las ventas digitales, (Guaña-Moya, et al. 2017). al analizar las tendencias globales de mercado se pueden observar e identificar cambios muy importantes en cuanto al comportamiento de los consumidores, en como acceden y consumen los propios medios de comunicación. (Ponce 2018).

\subsubsection{Generaciones de consumidores}

Según Jiménez 2018 hay 4 tipos de generaciones de consumo claramente diferenciadas. Estas son por orden cronológico:

1. Baby Boomers, estos son los nacidos entre 1945 y1964

2. Generación X, nacidos entre 1965 y 1980

3. Millennials, nacidos entre 1981 y 1994

4. Generación Z, desde 1995 hasta el 2010

Las tendencias digitales que se emplean con mayor frecuencia en cada una de las generaciones son; para los Baby boomers los medios preferidos son los correos electrónicos, la televisión, así como los medios impresos. En cuanto a las redes sociales empleadas con mayor frecuencia en esta generación son: Facebook, Twitter y LinkedIn. En segundo lugar, para la Generación X los medios en los que se mueven principalmente son el correo electrónico, las redes sociales (Facebook, twitter e Instagram) y la radio. En tercer lugar, para los Millennials ya se produce un cambio frente a las dos generaciones anteriores, ya que los medios en los que se encuentran son principalmente los digitales teniendo como redes sociales de referencia Facebook, YouTube e Instagram. Finalmente, para la Generación Z, se encuentran tanto en la televisión como en las redes sociales y las redes sociales preferidas por ellos son, YouTube Tik Tok e Instagram.

\subsubsection{Generación z y sus hábitos de consumo}


Esta generación es la primera que ha nacido íntegramente en una era digital ya generalizada. Esto ha hecho que cambie sus hábitos en medios digitales y de compra, con respecto a generaciones anteriores. para poder tener una visión más detallada a cerca de esta generación vamos a explicar algunos datos relevantes.

Según Bloomberg en el 2019 esta generación supero en numero a la anterior representando el 32\% de la población mundial. De este $32 \%$ de la población total el $74 \%$ pasan su tiempo libre empleando más de un dispositivo electrónico al mismo tiempo, que, a su vez, está conectado a la red. (Instituto de gestión empresarial 2017). El tiempo medio que pasan en sus dispositivos según datos del IAB 2020 es de 90 minutos al día de media, lo cual supone un incremento de 20 minutos diarios con respecto al año anterior.

Mientras que en todas las generaciones anteriores se asumía que la vida seria mejor que la de los antecesores, para los de la generación z saben que las opciones son algo más limitadas, lo cual hace que tengan una visión más pragmática y algo negativa del mundo que les rodea. Además, en cuanto a los hábitos de consumo el $72 \%$ considera que "tener cosas nuevas" está sobrevalorado si se satisfacen las necesidades. $Y$ el haber nacido en una época de crisis han hecho te adquieran un compromiso social más elevado que las generaciones antecesoras. Entender que es lo que impulsa y motiva a esta generación va a ser clave para conseguir el éxito de cualquier estrategia de comunicación. (Adglow 2018)

\subsubsection{Los influencers}

Con el surgimiento de la generación $z$, aparecen una serie de generadores de contenido que destacan por despertar interés a través de las redes sociales y en distintos grupos. Esta influencia la generan a través de su carisma, imagen que representan y credibilidad sobre aquello que representen. Estos influencers emiten opiniones sobre diferentes productos, servicios, empleando para comunicarse un lenguaje cercano a su publico objetivo, lo cual les genera cierta confianza y consiguen atraer su atención para que les sigan. (Fernandez de Bordóns \&Niño, 2021)

Los influencers surgen para realizar una comunicación más real y sincera con el fin de conectar con el consumidor. En 2021 siguen siendo un referente concretamente para los jóvenes, pues prestan más atención al mensaje prescrito por el influencer que al mensaje de la marca a través de un anuncio convencional. (Martín, 2021)

Gracias a esto, las marcas tratan de establecer relaciones comerciales con ellos con el fin de conectar mejor con su mercado y su publico, para que los influencers transmitan seguridad, transparencia y confianza a cerca de los diferentes productos o servicios que ofrezcan. (Ponce, 2018) 
Según H. Hatch (2012), en un estudio publicado por Harvard Bussiness, hay 5 características básicas que deben reunir os influencers para tener éxito. Estas características son:

1. Alcance: El alcance debe hacerse viral, llegando de los medios de comunicación tradicionales hasta los digitales.

2. Experiencia: hacer participes de sus experiencias a todos sus seguidores.

3. Credibilidad: ser transparentes para que su publico confíe en ellos.

4. Congregación: tener la capacidad de movilizar a los usuarios Confianza: generar una confianza y familiaridad con tus usuarios para que sientan más cercanía y que forman parte de tu vida.

\subsubsection{Marketing de influencers}

Hoy en día una de las estrategias de marketing más empleadas es el marketing de influencia ya que los usuarios confían más en las opiniones de otros usuarios (conocidos o no) que en campañas de publicidad tradicionales.

Las redes sociales son el lugar de ocio y entretenimiento de las últimas generaciones tal y como hemos comentado en puntos anteriores, por ello estas plataformas van a ser clave a la hora de definir una estrategia de marketing y de esa forma aumentar o mejorar el engagement que recibe una marca o producto. En este sentido, el contenido generado tendrá que ser auténtico, veraz y personalizado. (Gonzalez-Carrion \& Aguaded, 2020).

Un influencer en el ámbito de la moda tiene más probabilidad de conectar con seguidor de la generación $Z$, si muestra cómo le queda un vestido mientras sale de fiesta, o habla de una experiencia personal mientras se peina usando productos específicos para el cabello, ya que esta generación pone mucho más énfasis en las experiencias que ven a través de las redes sociales. Estrategias de esta índole podría conducir a una amplia fidelización en este segmento del mercado, forjando una fuerte conexión con estos consumidores. (Zuckerman, 2019)

El papel de los influencers está creciendo y cambiando constantemente a medida que más generación $\mathrm{Z}$ y Millennials utilizan las redes sociales con fines distintos a la comunicación. Las principales funcionalidades e intereses para las mujeres son la belleza, moda, comida y viajes, mientras que para los hombres son, juegos, deporte, comida y viajes, según el informe de influencers del 2019.

Ahora, la comunicación está dominada por el presentismo y por lo efímero (reels, instastories, directos...). El marketing de moda ha evolucionado en el ámbito digital hasta el punto de que la producción y venta de los productos esté concebida para ser instagrameable, desde campañas a desfiles y diseños. (Velasco, 2021)

Cada vez son más las marcas que comprenden los beneficios del marketing de influencers y aprovechan esta salida en su beneficio. Dado que los jóvenes de hoy en 
día se rodean digitalmente de influencers para aprender sobre diversos temas y productos, los profesionales del marketing tienen que fortalecer sus estrategias de medios sociales con las asociaciones de influencers adecuados si esperan llegar de manera efectiva a la próxima generación de consumidores.

El éxito de este tipo de marketing se ha convertido en una estrategia esencial para algunas empresas, ya que además de generar ventas, también producen mayor cercanía y credibilidad con la marca. Y tal y como dice Adglow, los principales objetivos de las marcas son: conseguir alcance (36,8\%), visualizaciones de vídeos $(30,7 \%)$, obtención de clicks en los enlaces de sus anuncios (18,9\%), engagement $(10 \%)$ y conversiones $(1,80 \%)$.

Aun así, según datos del IAB del 2020, el seguimiento de influencia este año ha disminuido con respecto al año anterior, siendo Instagram la red social en la que más se sigue a influencers, tal y como podemos observar en la gráfica 1.

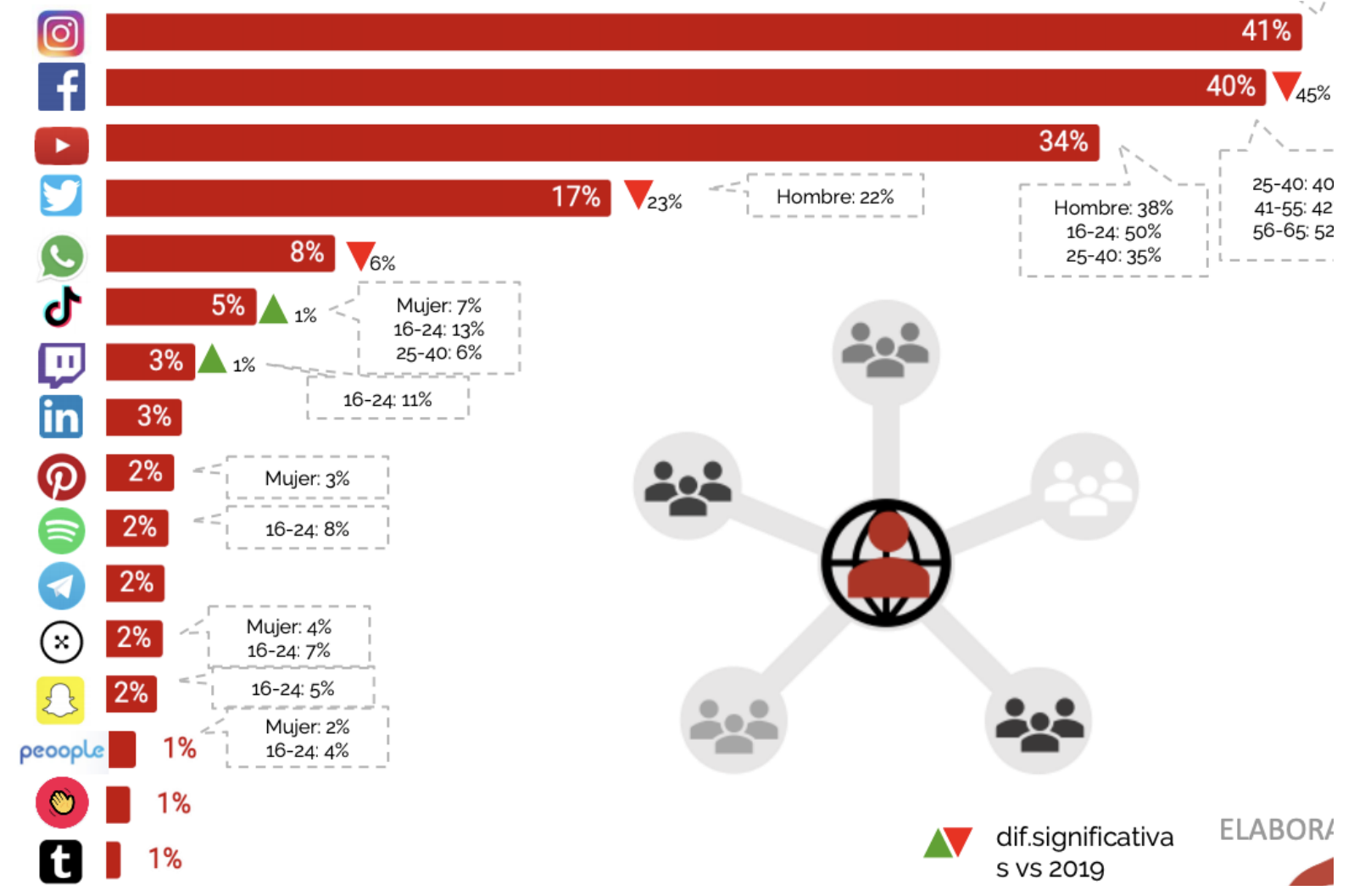

Foto 1. Porcentaje de internautas que siguen a influencers en las redes sociales en España en 2020

Fuente: Elaboración propia

\subsection{Estudio de campo}


La encuesta analizada se ha basado en un tipo de investigación cualitativa realizada a través de Google Forms. compartida mediante Instagram y Whatsapp a los jóvenes pertenecientes a la generación Z entre el 21 y el 24 de noviembre del 2020.

La investigación en cuestión se ha centrado en los hábitos de consumo de moda mediante tendencias comunicativas como la publicidad en redes sociales y la realizada mediante figuras como los influencers.

El target de la encuesta realizada ha sido la generación Z. Esta está formada por personas que se encuentran entre los 10 y los 25 años. Como resultado de la investigación, se han recopilado 500 respuestas de las cuales el $77,6 \%$ han sido proporcionadas por mujeres y el resto por hombres. El $43,6 \%$ de los encuestados tienen entre 20 y 22 años, el $26,9 \%$ entre 23 y 25 años, el $15,4 \%$ entre 10 y 16 y el resto entre 17 y 19 años.

\subsubsection{Objetivos}

El objetivo principal de la encuesta ha sido investigar cuáles son los hábitos de consumo de moda dentro de los jóvenes que se encuentran entre los 10 y los 25 años teniendo en cuenta la repercusión de la figura de los influencers y su eficacia.

Para lograr un análisis más completo, además de entender la mentalidad de la generación $\mathrm{Z}$ respecto a las preferencias por las herramientas en publicidad de moda y su efectividad, se han tenido en mente objetivos secundarios como los medios, el contenido, los gustos y las marcas de moda preferidas por los consumidores.

\subsubsection{Planificación y diseño de la investigación}

Tras realizar varios modelos y diversas modificaciones en la encuesta para centrar el tema, además de definir los objetivos, surgió la encuesta final, siendo esta validada por tres expertos en investigación de mercados. Las primeras preguntas realizadas son tipo multirespuesta para poder obtener los datos sobre las preferencias de los consumidores. A medida que la encuesta va avanzando, las preguntas empiezan a ser más especificas con el objetivo de buscar justificación a las acciones de los encuestados para finalmente llegar a las conclusiones.

La encuesta está compuesta por varias imágenes para que resulte más atractiva visualmente. Las respuestas son de diferentes formatos como de cuadrículas de diferentes opciones, de opción de respuesta, de respuesta predeterminada, de respuesta corta o de escala lineal para que no sean repetitivas y resulten más dinámicas.

\subsubsection{Análisis e interpretación de los resultados}

Teniendo en cuenta la primera gráfica, cabe destacar la preferencia de los encuestados por la red social de Instagram con un 90,4\% frente a los demás medios a 
la hora de ver publicidad relacionada con la moda. Tanto la televisión como la publicidad exterior ocupan el segundo lugar con un $48,1 \%$ y Youtube ocupa el tercer lugar con un $48.1 \%$ seguido por series/cine con un $32,1 \%$. En cambio, redes sociales como email, Facebook poseen porcentajes por debajo del $2 \%$ del total.

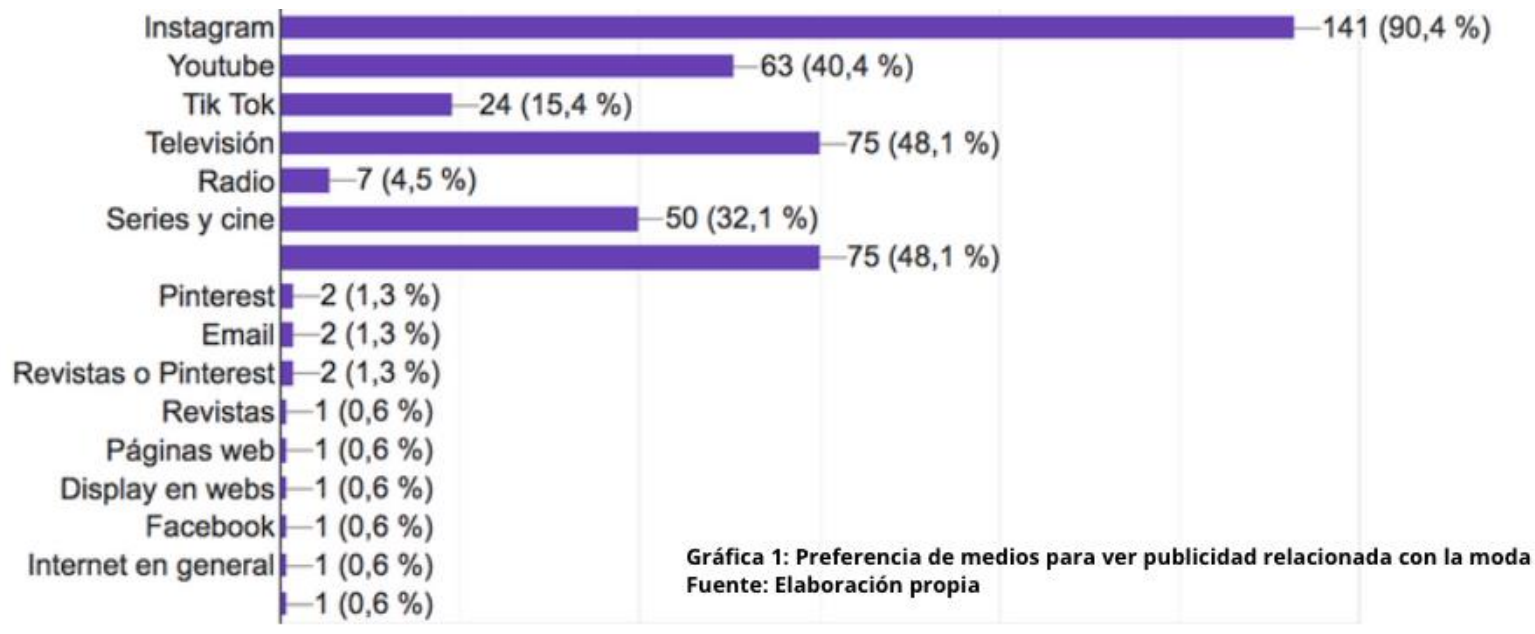

Gráfica 1. Preferencia de medios para ver publicidad relacionada con la moda.

Fuente: Elaboración propia

La gran parte de los encuestados justifican que los medios mencionados son los más adecuados para ver publicidad relacionada con la moda debido a que son los que más utilizan a diario además de ser los canales más visuales, directos y atractivos. Se repiten respuestas que afirman que la publicidad no les molesta en redes como Instagram o Youtube a diferencia de en otros medios.

Teniendo en cuenta las nuevas herramientas de las redes sociales cabe destacar el último apartado de Instagram "tienda". Como se puede observar en la gráfica 2, este resulta ser útil a la hora de comprar productos para la mayoría de los usuarios de esta aplicación con un porcentaje del 52.3\%. Además, otras herramientas de las redes como Instastories y Reels en caso de Instagram o videos de Tik Tok son más efectivas cuando se trata de publicitar una marca. Esto se ve reflejado en la siguiente gráfica mediante la respuesta del $56.4 \%$ de los encuestados. 
Martín Critikián, D., Solano Altaba, M., y Serrano Oceja, J. F.

Hábitos de consumo de moda a través de influencers en la Generación Z
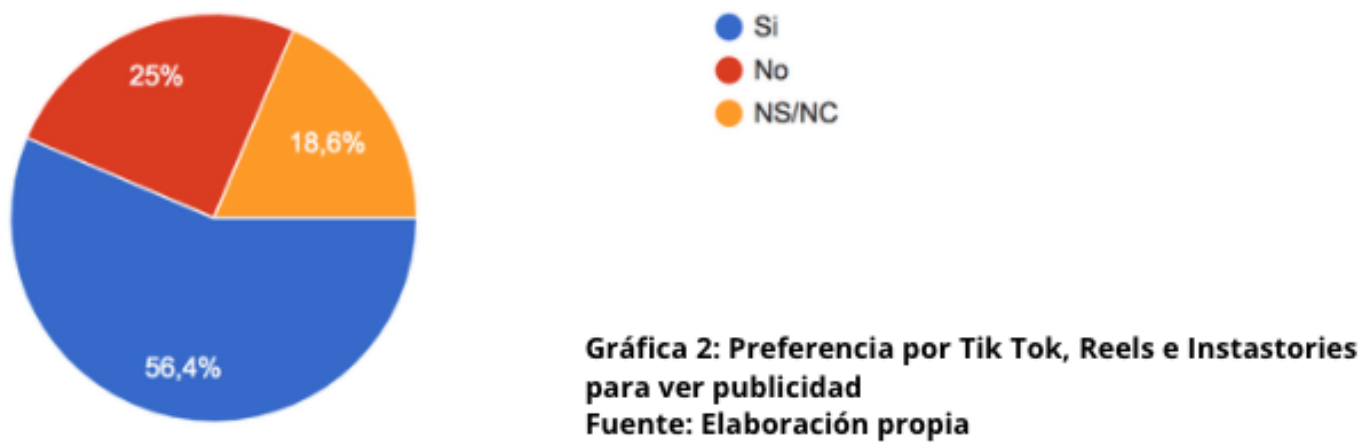

Gráfica 2. Preferencia por Tik Tok, Reels e Instastories para ver publicidad

Fuente: Elaboración propia

En cuanto al contenido de la publicidad destaca con una gran diferencia, la preferencia de los consumidores por los anuncios breves y originales, que tan solo muestren el producto y la marca. La mayoría de los consumidores consideran que la cualidades, el precio, la calidad y la marca del producto los son los factores que la publicidad tiene que mostrar. Los anuncios emocionales que transmiten la experiencia con el producto son los segundos más seleccionados con un 17,2\%.
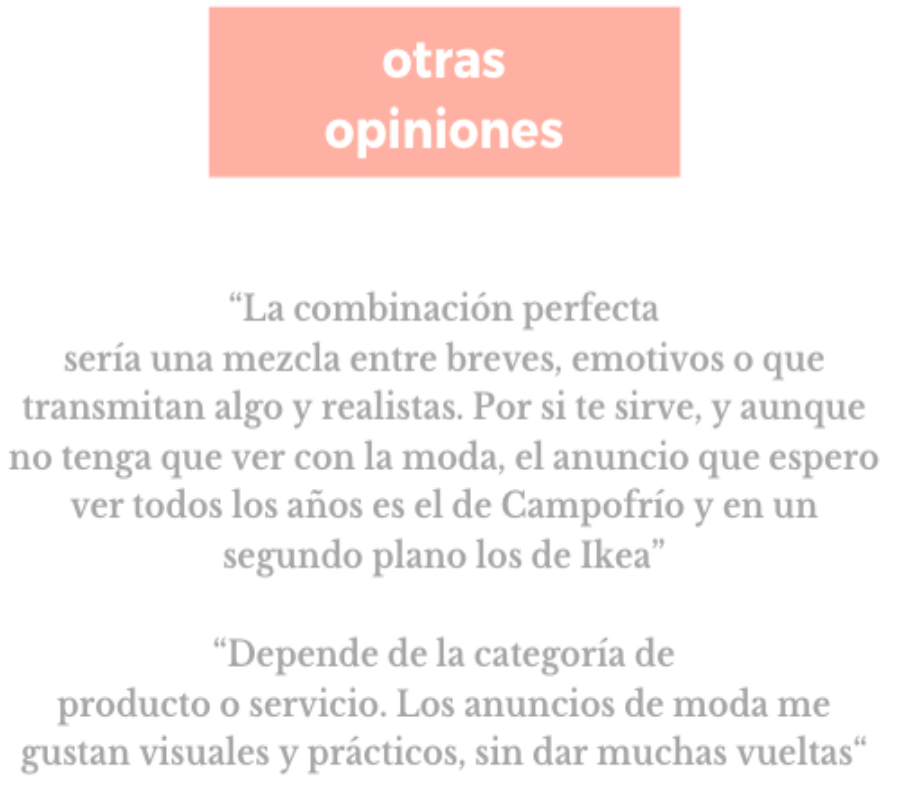

Foto 2. Otras opiniones

Fuente: Elaboración propia

Los datos relacionados con la repercusión que tienen los influencers en los hábitos de consumo de los usuarios de redes sociales de la gráfica 3, muestran de una forma clara que estos influido a la mayoría de ellos. El 37,8\% afirma que esto se debe al 
hecho de que los influencers muestran todas las características y funcionalidades de los productos publicitados. El 21,3\% afirma que se debe a que anticipan lo que va a estar de moda. En cambio, el 17,3\% del porcentaje restante admite que no se fían de sus recomendaciones.

Haciendo referencia al posicionamiento de las marcas el sector de la moda, cabe destacar que tal y como muestra la gráfica 4, el 93,6\% de los encuestados afirman que han descubierto nuevas marcas gracias a las menciones o a las etiquetas en las redes sociales de influencers. El 54,2\% del total tiene preferencia por la publicidad que se inspira en looks de influencers para recrearlos con productos low cost. Marcas reconocidas como Nike, Zara, Name the Brand o Pull \& Bear son las más mencionadas por los encuestados teniendo en cuenta las marcas de moda que consideran que realizan una buena publicidad. Name the Brand, Pura vida y Noon también son mencionadas entre otras.
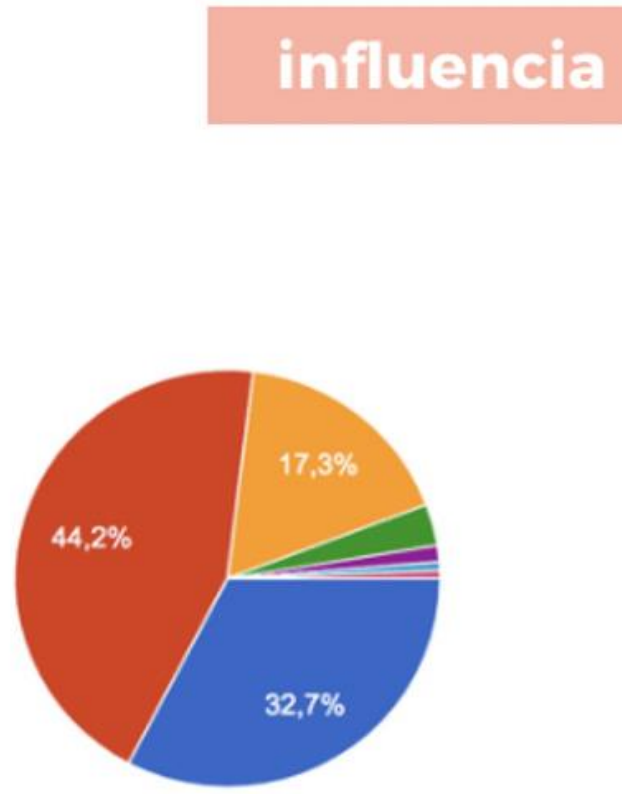

Si, en diversas ocasiones.

Si pero con poca frecuencia.

No, no me fio de sus recomendaciones.

NS/NC

No

No. No es que no me fie, sino que sé que es publicidad pagada y no lo publicitan porque realmente les guste.

No, pero no porque no me fie

\section{Gráfica 3: Frecuencia de la influencia de personajes populares para comprar Fuente: Elaboración propia}

Gráfica 3. Frecuencia de la influencia de personajes populares para comprar.

Fuente: Elaboración propia 
Martín Critikián, D., Solano Altaba, M., y Serrano Oceja, J. F.

Hábitos de consumo de moda a través de influencers en la Generación Z
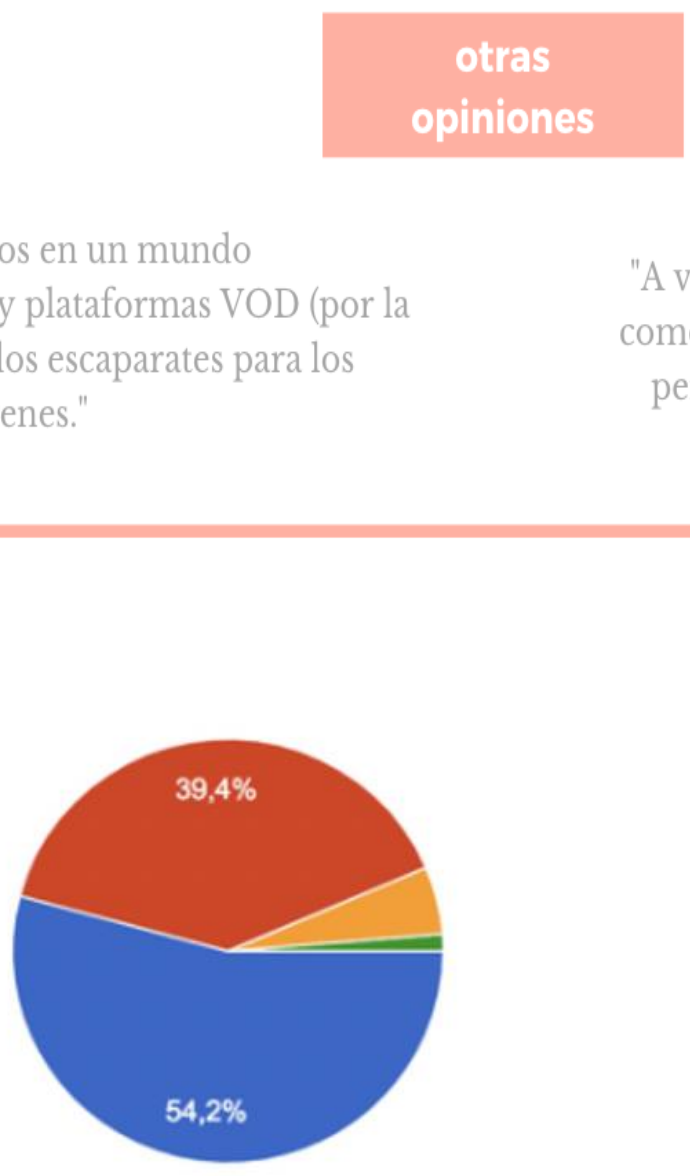

Gráfica 4: Cantidad de marcas conocidas mediante las redes sociales de los influencers Fuente: Elaboración propia

Gráfica 4. Cantidad de marcas conocidas mediante las redes sociales de los influencers.

Fuente: Elaboración propia

Por último, tras el análisis e interpretación de los resultados, se podría decir que teniendo en cuenta la situación y preferencias de la generación $Z$, se identifica que tanto las marcas relacionadas con la moda, como la publicidad que realizan las 
mismas, deben ser más directas y compartidas en más medios teniendo como referencia Instagram para ser más eficaces y fomentar la compra de productos entre los jóvenes de 10 a 25 años. Las figuras como la de los influencers brindan a la marca la oportunidad de mostrar los productos como si de un escaparate se tratase. Con lo cual, cuanto más se comparta esa publicidad en diferentes perfiles, más veces se visualizará el producto por diversos consumidores potenciales, logrando como resultado la influencia en los hábitos del consumo de estos.

Foto 3. Otras opiniones

Fuente: Elaboración propia

\subsubsection{Conclusiones de la investigación}

Instagram es el medio por excelencia para la generación $\mathrm{Z}$ a la hora de ver publicidad relacionada con la moda, seguido por Televisión/ publicidad exterior y Youtube debido a que son canales prácticos, dinámicos, directos y visuales.

Los videos cortos como reels o tik toks y las herramientas como "tienda" de Instagram son más eficaces para publicitar productos relacionados con la moda debido a que los jóvenes prefieren anuncios breves y originales que muestren la marca.

A pesar de que la generación $\mathrm{Z}$ niegue que las redes sociales fomentan su consumo de productos, la mayoría admite haber comprado productos además de conocer marcas relacionadas con la moda debido al impacto de los influencers.

Los influencers tienen poder sobre los hábitos de consumo de la generación $\mathrm{Z}$ debido a que muestran las funcionalidades de los productos además de anticipar los productos que van a estar de moda.

Las marcas visualizadas en diferentes medios y perfiles son más valoradas debido a que la reputación y la imagen de estas es un factor importante a la hora de posicionarlas en la mente de los consumidores de la generación $\mathrm{Z}$.

\section{DISCUSIÓN}

Tras haber realizado una revisión blibliográfica exhaustiva y la investigación de campo realizada sobre los hábitos de consumo de la generación $\mathrm{Z}$, nos llevan a concluir que son una generación global y social, que ha vivido una crisis económica nacional y que se encuentra en la era de la sobreinformación. Son 100\% nativos digitales, multipantallas y multitareas. Es una generación que emplea una media de entre 1 y 3 horas diarias a las redes sociales, lo que afecta a diferentes áreas de su vida, entre las que podemos destacar sus relaciones personales (amigos, familiares...) o su estado de ánimo y autoestima. 
Teniendo en cuenta la situación y preferencias de la generación $Z$, se identifica que tanto las marcas relacionadas con la moda, como la publicidad que realizan las mismas, deben ser más directas y compartidas en más medios teniendo como referencia Instagram para ser más eficaces y fomentar la compra de productos entre los jóvenes de 10 a 25 años.

Las figuras como la de los influencers brindan a la marca la oportunidad de mostrar los productos como si de un escaparate se tratase. Con lo cual, cuanto más se comparta esa publicidad en diferentes perfiles, más veces se visualizará el producto por diversos consumidores potenciales, logrando como resultado la influencia en los hábitos del consumo de estos. Estrategias de personificación de la experiencia podría conducir a una amplia fidelización en este segmento del mercado, forjando una fuerte conexión con estos consumidores.

Según los datos presentados, el papel de los influencers está cambiando constantemente a medida que la generación $\mathrm{Z}$ utiliza las redes sociales con fines distintos a la comunicación. Las principales funcionalidades e intereses para las mujeres son la belleza, moda, comida y viajes, mientras que para los hombres son, juegos, deporte, comida y viajes, según el informe de influencers del 2019. Los influencers tienen poder sobre los hábitos de consumo de la generación $\mathrm{Z}$ debido a que muestran las funcionalidades de los productos además de anticipar los productos que van a estar de moda.

\section{CONCLUSIONES}

Como se ha podido comprobar, esta generación autentica, comprometida, digital, multipantalla y multitarea, no confían en la publicidad a través de medios de comunicación tradicionales, sino que prefieren una persona de carne y hueso con la que se sientan identificados.

A pesar de que a la Generación $Z$ se identifique con los influencers, en este año 2020 según el informe del IAB, la eficiencia del marketing de influencers disminuyo un $41 \%$ en comparación con los resultados obtenidos del año anterior, aunque las campañas realcionadas con el cuidado de la salud, la alimentación y los bienes de consumo doméstico, jardinería, el deporte, las manualidades o los juegos de azar, que crecieron notablemente.

Por otro lado, Bluemedia Studio, nos desvela que los grandes influencers (más de 50.000 seguidores) generan menor interacción que los nanoinfluencers (menos de 10.000 seguidores) o los microinfluencers (entre 10.000 y 50.000 seguidores), llegando a copar hasta un $40 \%$ de las asociaciones con marcas, que encuentran en estos perfiles un público más fiable y leal. (Bluemedia Studio 2020)

En cuanto a los hábitos de consumo de la Generación Z, Instagram es el medio por excelencia a la hora de ver publicidad relacionada con la moda, seguido por 
Televisión/ publicidad exterior y Youtube debido a que son canales prácticos, dinámicos, directos y visuales.

Los videos cortos como Reels o Tik Toks y las herramientas como "tienda" de Instagram son más eficaces para publicitar productos relacionados con la moda debido a que los jóvenes prefieren anuncios breves y originales que muestren la marca. Son una genercion que la prioridad es disfrutar más que tener.

Si algo es característico de esta generación es su habilidad para compartir bienes, servicios, conocimiento, opiniones, ya no piensan en el "yo" sino que prevalece el compartir. Por ello, entienden, apoyan y aceptan la economía colaborativa en la que intervienen activamente, como consumidores. (Lara 2017)

\section{REFERENCIAS}

Atrevia. (2016). Informe Generación Z: el último salto generacional. https:// www.atrevia.com/blog/informe-generacion-z-el-ultimosaltogeneracional/

Bell, D. (2001). El advenimiento de la sociedad post-industrial. Un intento de prognosis social. Madrid: Alianza Editorial

Bluemedia Studio. (2020). ¿Cómo influyen realmente los 'influencers' en nuestros hábitos de consumo?. La información. https:// www.lainformacion.com/brandedcontent/estar-donde-estes/como-influyen-realmente-los-influencers-en-nuestroshábitos-de-consumo/2818421/

Castells, M. (2000). La era de la información: economía, sociedad y cultura. Madrid: Alianza Editorial, S.A.

Castells, M. (2012). Redes de Indignación y Esperanza. Madrid: Alianza.

Calderón, N. (s.f.) La socialización como elemento fundamental en la vida. www.psicopedagogia.com

Cerón, N. (2015) Concepto de internet. http://atotonilco1103nayel.blogspot.com.es/2015/11/resultado-de-imagen-paraimagenes- de.html

Cornellá, A. (1998) ¿Economía de la información o Sociedad de la información? ESADE

Barcelona. http://www.ccee.edu.uy/ensenian/catcomp/material/doc2eco.pdf

Drucker, P. (1996). Landmarks of Tomorrow. New York: Harper. 
Martín Critikián, D., Solano Altaba, M., y Serrano Oceja, J. F.

Hábitos de consumo de moda a través de influencers en la Generación Z

Farnos, J. D. (s.f). La sociedad del conocimiento: las tic y su influencia social y educativa. https://juandomingofarnos.wordpress.com/2011/09/12/la-sociedaddel- conocimiento-las-tic-su-influencia-social-y-educativa/

Fernandez, D. V. (2019). La generación Z: el objetivo principal del nuevo marketing en redes sociales mediante influencers de moda.

Fernández De Bordóns, B., \& Niño González, J. I. (2021). Análisis del consumidor en redes sociales y su percepción de las marcas. Revista De Comunicación De La SEECI, (54), 21-54. https://doi.org/10.15198/ seeci.2021.54.e628

Finquelievich, S. (2008). La Sociedad de la Información: ¿una nueva disciplina científica?. Revista Iberoamericana de Ciencia, Tecnología y Sociedad. http://www.oei.es/historico/divulgacioncientifica/opinion0063.htm

Foray, D. (2002). La sociedad del conocimiento. Revista Internacional de Ciencias sociales, 171.

Gonzalez-Carrion, E. L., \& Aguaded, I. (2020). Engagement y evolución de instagramers hispanohablantes de moda . Revista Latina De Comunicación Social, 77, 231-252. https://doi.org/10.4185/RLCS-2020-1456

Gómez, J. A., (1999). Propuesta de Directiva sobre determinados aspectos de los derechos de autor y los derechos afines en la sociedad de la información, Madrid: Editorial Reus S.A.

Guaña- Moya, E. J., Quinatoa-Arequipa, E., \& Perex-Fabara, M. A. (2017). Tendencias del uso de las tecnologías y conductas del consumidor tecnológico. Ciencias Holguín

Hatch, H. (2012). Influenciadores ¿Quiénes con realmente? http://www.merca20.com/influenciadores-quienes-son-realmente/

Institute for Business Value. (2017). Uniquely Generation Z https://www.generationy20.com/retail-generation-z.PDF

Influencer Marketing Hub. (2019). El Estado del Marketing de Influencers 2019: Informe Benchmark. https://influencermarketinghub.com/es/informebechmark-marketing-de-influencers-2019/

Krüger, K. (2006). El concepto de sociedad del conocimiento. Revista bibliográfica de geografía y ciencias sociales (serie documental de Geo Crítica). 683 (XI), Universidad de Barcelona. http://www.ub.edu/geocrit/b3w- 683.htm

Lara, I. (2017). Los consumidores de la Generación Z impulsan la transformación digital de las empresas. Revista estudios de juventud. 
Martín Critikián, D., Solano Altaba, M., y Serrano Oceja, J. F.

Hábitos de consumo de moda a través de influencers en la Generación Z

http://www.injuve.es/sites/default/files/2017/28/publicaciones/documentos_5 ._los_consumidores_de_la_generacion_z.pdf

Maestre, J. V. (2007). Los medios de comunicación y el proceso de aprendizaje. Le Monde

http://www.aldeaglobal.net/jvmestre/medioscomunicacion.html

Diplomatic.

Martín, A. (2021). Percepción de los estudiantes del grado de publicidad y RR.PP. Sobre la relación entre marcas e influencers en Instagram. Vivat Academia. Revista de

Comunicación.

https://www.vivatacademia.net/index.php/vivat/article/view/1337/2161

Martinez, J. A, Lara- Navarra, P. y Beltrán, P. (2006). La influencia de la sociedad del conocimiento en la modernización de la Administración Pública. uocpapers, No. 3 http://www.uoc.edu/uocpapers/3/dt/esp/martinez_lara_beltran.pdf

Miller, L. (2018). Gen Z Is Set to Outnumber Millennials Within a Year. Bloomberg. https://www.bloomberg.com/news/articles/2018-08-20/gen-z-to-outnumbermillennials-within-a-year-demographic-trends

Ponce, C. E. (2018). Como los influencers han cambiado los hábitos de consumo de los millennials en Guayaquil. https://dspace.ups.edu.ec/bitstream/123456789/15535/1/UPS-GT002122.pdf

Prats, J. (2015) La población en riesgo de pobreza o exclusión social crece hasta el $29 \%$

http://economia.elpais.com/economia/2015/05/26/actualidad/1432626857_8093 10.html

Real Academia de la Lengua Española. http://dle.rae.es/?id=KzwDY4y

Sequeiros, C., Puente, H., López, M., \& Fernández, M. (2016). Juventud, cultura y NTICs: ¿Triángulo o prisma? Teknokultura, 711.

Sinay, S. (2009). La sociedad de los hijos huérfanos. Ediciones B.

Szabo, C. (2005). Knowledge-based society?. University of Miskolc. http:// www.unimiskolc.hu/uni/res/kozlemenyek/2005- 2/pdf/szabocs.html

Velasco, A. M. (2021). Influencers, storytelling y emociones: marketing digital en el sector de las marcas de moda y el lujo. Vivat Academia. Revista de Comunicación. https://www.vivatacademia.net/index.php/vivat/article/view/1321/2155

Villegas, C. A. (2010). Fundamentos socioculturales de la educación. E-Historia. http://www.e-historia.cl/e-historia/fundamentos-socioculturales-de-laeducacion/ 
Zuckerman, M. (2019). Influencer Marketing: Millennials y Centennials. https://blog.digimind.com/es/insight-driven-marketing/author/melissazuckerman

\section{AUTORES:}

\section{Davinia Martín Critikián}

Apasionada de la formación, comunicación y las redes sociales, trabaja desde el 2017 como profesora colaboradora doctora en la Universidad CEU San Pablo y del 2011 al 2017 en la Universidad Internacional de La Rioja. Interés en seguir desarrollando mi faceta investigadora y docente en la cual se han publicado numerosos capítulos de libros y participado en un proyecto precompetitivo y en un grupo de investigación consolidado.

Orcid ID: https:/ / orcid.org/0000-0002-6921-4707

\section{María Solano Altaba}

Decana de la Facultad de Humanidades y Ciencias de la Comunicación de la Universidad CEU San Pablo. Periodista en ejercicio y doctora en periodismo, ha centrado sus líneas de investigación en la posverdad, las fake news, la alfabetización mediática entre los jóvenes y las transformaciones de las rutinas periodísticas y publicitarias en el entorno digital.

Orcid ID: https://orcid.org/0000-0002-1489-0695

\section{José Francisco Serrano Oceja}

Doctor en Ciencias de la Información, profesor titular acreditado por la ANECA, es autor de varios libros y de medio centenar de artículos de investigación sobre Periodismo y Comunicación. Sus líneas de investigación prioritaria son la retórica trasmedia, el periodismo especializado y las gnoseologías de la información. Profesor de varios Másters, es académico invitado en varias universidades de América Latina.

Orcid ID: $\underline{\text { https://orcid.org/0000-0001-7364-7786 }}$ 